BMJ Open

Diabetes

Research

\& Care

\title{
Time in range-A1c hemoglobin relationship in continuous glucose monitoring of type 1 diabetes: a real- world study
}

\author{
Marina Valenzano (D) , ${ }^{1}$ Ivan Cibrario Bertolotti (D) , ${ }^{2}$ Adriano Valenzano (D) , \\ Giorgio Grassi ${ }^{3}{ }^{3}$
}

To cite: Valenzano M, Cibrario Bertolotti I, Valenzano A, et al. Time in range-A1c hemoglobin relationship in continuous glucose monitoring of type 1 diabetes: a real-world study. BMJ Open Diab Res Care 2021;9:e001045. doi:10.1136/ bmjdrc-2019-001045

- Supplemental material is published online only. To view please visit the journal online (http://dx.doi.org/10.1136/ bmjdrc-2019-001045).

Received 14 November 2019 Revised 21 May 2020 Accepted 10 January 2021

Check for updates

(C) Author(s) (or their employer(s)) 2021. Re-use permitted under CC BY-NC. No commercial re-use. See rights and permissions. Published by BMJ.

${ }^{1}$ Division of Endocrinology and Metabolic Diseases, Department of Medical Sciences, University of Turin, Torino, Piemonte, Italy

${ }^{2}$ Institute of Electronics, Information and Telecommunication Engineering, CNR IEIIT, Torino, Piemonte, Italy

${ }^{3}$ Endocrinology, Ospedale Molinette, Torino, Piemonte, Italy

Correspondence to Dr Giorgio Grassi; giorgio.grassi@gmail.com

\section{ABSTRACT}

Introduction The availability of easily accessible continuous glucose monitoring (CGM) metrics can improve glycemic control in diabetes, and they may even become a viable alternative to hemoglobin $\mathrm{A} 1 \mathrm{c}(\mathrm{HbA1c})$ laboratory tests in the next years. The REALISM-T1D study (REAl-Life glucoSe Monitoring in Type 1 Diabetes) was aimed at contributing, with real-world data, to a deeper understanding of these metrics, including the time in range (TIR)-HbA1c relationship, to facilitate their adoption by diabetologists in everyday practice.

Research design and methods 70 adults affected by type 1 diabetes were monitored for 1 year by means of either flash (FGM) or real-time (rtCGM) glucose monitoring devices. Follow-up visits were performed after 90, 180 and 365 days from baseline and percentage $\operatorname{TIR}^{70-180}$ evaluated for the 90-day time period preceding each visit. $\mathrm{HbA} 1 \mathrm{C}$ tests were also carried out in the same occasions and measured values paired with the corresponding TIR data. Results A monovariate linear regression analysis confirms a strong correlation between $\mathrm{TIR}$ and $\mathrm{HbA1C}$ as found in previous studies, but leveraging more homogeneous data $(n=146)$ collected in real-life conditions. Differences were determined between FGM and rtCGM devices in Pearson's correlation $\left(r_{\mathrm{FGM}}=0.703, r_{\text {rtCGM }}=0.739\right)$, slope $\left(\beta_{1, \mathrm{FGM}}=-11.77, \beta_{1, \mathrm{rCGM}}=-10.74\right)$ and intercept $\left(\beta_{0, \mathrm{FGM}}=141.19, \beta_{0, \mathrm{rCGM}}=140.77\right)$ coefficients. Normality of residuals and homoscedasticity were successfully verified in both cases.

Conclusions Regression lines for two patient groups monitored through FGM and rtCGM devices, respectively, while confirming a linear relationship between TIR and A1c hemoglobin (A1C) in good accordance with previous studies, also show a statistically significant difference in the regression intercept, thus suggesting the need for different models tailored to device characteristics. The predictive power of $\mathrm{A} 1 \mathrm{C}$ as a TIR estimator also deserves further investigations.

\section{INTRODUCTION}

Continuous glucose monitoring (CGM) has opened new horizons in diabetes care, ${ }^{1-3}$ enabling frequent measurements of blood glucose through minimally invasive techniques and wearable devices. ${ }^{4}$ Moreover,

\section{Significance of this study}

What is already known about this subject?

- Continuous glucose monitor (CGM)-derived metrics, like $\operatorname{TIR}^{70-180}$, are easily accessible, but users need to become more familiar with their interpretation in order to optimize clinical outcomes.

- A good understanding of the relation between time in range (TIR) and the biomarker hemoglobin A1c $(\mathrm{HbA1C})$, which is still the gold standard for clinical judgment on glycemic control, is crucial if TIR is to complement HbA1c or become a viable alternative to it in the next years.

What are the new findings?

- Results confirm a strong linear correlation between HbA1C and TIR based on 90-day CGM data in reallife conditions, whereas quadratic contributions reported in other studies were found to be negligible.

- The work revealed a statistically significant difference in the regression intercept of flash (FGM) and real-time (rtCGM) sensor data and highlighted that tailored models are likely to be required for different monitoring systems; statistical significance tests performed on the regression slope difference were instead inconclusive.

How might these results change the focus of research or clinical practice?

- CGM-related metrics might provide valuable and actionable information not just for instant overview but also for long-term disease management.

- Clinical judgment based on CGM metrics should also take into account differences in devices and relevant TIR-HbA1c relationships.

CGM makes available to diabetologists parameters and metrics, ${ }^{67}$ such as time in range (TIR), time above range, time below range and coefficient of variation, which can be leveraged in effective glucose control and might prevail over, although not replace, A1c hemoglobin (A1C) laboratory tests in the years to come. ${ }^{8-10}$ On the one hand, the 
adoption of the A1C biomarker has been well assessed in the usual clinical practice for quite a long time and is currently the gold standard for follow-up in metabolic control or diabetes outcomes assessment. A1C reflects hyperglycemia and its association with chronic disease complications, but unfortunately has also proven to be unsuitable for assessing daily glycemic variations ${ }^{11} 12$ and in particular determining short-term glycemic changes accurately. ${ }^{13}$ Thus, further tailored evaluations are needed when dealing with individual care and in the attempt to realize precision medicine. ${ }^{14}$ On the other hand, CGM systems are both gaining popularity and being increasingly adopted thanks to fast advances in sensor and device technology. ${ }^{51516}$

TIR is a recognized key metric readily available for targeting insulin therapy day by day. Thus, it is more flexible and provides more actionable information than A1C alone. However, evidence regarding correlation with long-term glycemic control and diabetes complications is limited and will probably require years to collect. Thus, clinicians could feel more confident in targeting patients' glucose management according to the new indicator if a strong correlation with the gold standard (A1C) was confirmed. Percentages of desirable 'time in range' might also be refined, depending on observed relationship with $\mathrm{A} 1 \mathrm{C}$ values, until a reliable prediction of $\mathrm{A} 1 \mathrm{C}$ levels is possible.

Some recent retrospective and cohort studies ${ }^{17-19}$ have focused on the existing relationships between CGM metrics and $\mathrm{A} 1 \mathrm{C}$ to assess their correspondences and differences. Moreover, real-world observational studies are gaining regard as an additional source of information that can help in better understanding findings of clinical trials. $^{20} 21$

A real-world observational study, called 'REAl-Life glucoSe Monitoring in Type 1 Diabetes' (REALISM-T1D), was conducted in Torino, Italy, to gain more insight on emerging CGM-enabled metrics in diabetes care and in particular the relationship between percentual time-inrange $70-180 \mathrm{mg} / \mathrm{dL}$ and $\mathrm{A} 1 \mathrm{C}$. The study was carried out jointly by two Italian institutions: the Department of Medical Science, University of Turin, and the Institute of Electronics, Computer and Telecommunications Engineering (IEIIT) of the National Research Council of Italy (CNR).

\section{RESEARCH DESIGN AND METHODS}

\section{Study design and participants}

REALISM-T1D was based on real-world observations of adult patients with type 1 diabetes who did not have any previous experience with CGM; they were monitored for a full 12-month period in their daily life through commercial CGM devices. The study involved a single diabetes care center at 'Le Molinette' hospital in Torino, which is not devoted to the treatment of young (under 18) patients with diabetes as they are addressed to different facilities. Every year about 100 real-time (rtCGM) and flash (FGM) sensors are prescribed for the first time to adults with type 1 and type 2 diabetes who refer to the center, while about 4000 patients are currently receiving follow-up visits and treatment.

A total of 70 adult patients of Caucasian race with type 1 diabetes participated in the study. In particular, 94 individuals aged 20-60 were screened, who turned to the center for the first time in a 12-month time period (between April 1, 2017 and March 31, 2018) and did not have previous experience with CGM. Eleven did not qualify, while 13 did not consent to participate. Eventually, 70 patients were enrolled depending on their voluntary informed consensus to be included in the investigation, with a mean age of $40.4 \pm 12.9$ years (range 20-60), disease duration of $21.6 \pm 14.0$ years (range $1-54$ ), body mass index (BMI) of $24.8 \pm 3.5 \mathrm{~kg} / \mathrm{m}^{2}$ and $\mathrm{A} 1 \mathrm{C}$ of $7.6 \% \pm 1.0 \%(60 \pm 10 \mathrm{mmol} / \mathrm{mol})$. To grant personal data protection, pseudonymization was used and transcoding tables were stored in a single protected server of the care center. In the selection, no constraint was imposed on the metabolic compensation in terms of $\mathrm{AlC}$ at the baseline and stable complications. Instead, the only reasons for exclusion were the presence of severe complications at an advanced stage, uncompensated psychiatric disorders, and dangerous societal problems sometimes encountered in a negligible minority of the center patients. Differently from randomized controlled trials, there was no need for a control group equipped with blinded devices, while inclusion/exclusion criteria, such as patients already at the target with $\mathrm{A} 1 \mathrm{C}$ under $7 \%(53 \mathrm{mmol} / \mathrm{mol})$, could be avoided.

Patients were first given instructions about the use of the prescribed devices and the procedures to calibrate their sensors and upload CGM data (in the case they were requested to do so themselves, in particular with rtCGM sensors) during a preliminary visit and a subsequent 2-week training, and then CGM was started and data seamlessly collected for a full 1-year time period. As the study was purely observational by design, participation had no influence on the behavior and decision of the care team and patients were let free to manage their devices in their everyday glucose monitoring. Insulin therapies, however, resulted evenly distributed between multiple daily injections and insulin pumps.

Three follow-up visits were planned at 90, 180, and 365 days from the beginning of the monitoring activity, and on those occasions A1C laboratory tests were also performed based on the high-performance liquid chromatography (HPLC) ion-exchange chromatography method. Sensor data were uploaded to the relevant websites by either the patients themselves at home or the center physicians during the follow-up visits. Observations were successfully completed at the end of March 2019 for 59 patients out of the 70 belonging to the initial pool. The group at the end of the study consisted of patients with a mean age of $41.4 \pm 13.1$ years (range 21-61), disease duration of $22.6 \pm 14.3$ years (range 2-55), BMI of $24.2 \pm 3.8 \mathrm{~kg}$ / $\mathrm{m}^{2}$ and $\mathrm{AlC}$ of $7.2 \% \pm 0.9 \%(55 \pm 10 \mathrm{mmol} / \mathrm{mol})$. The 11 
dropouts were caused by patients not reporting sensor faults promptly (two cases), incorrect sensor management for more than $30 \%$ of the monitoring timeframe (seven cases) and patients moving to a different region and care center (two cases).

Only commercial monitoring devices whose cost is repayable by the Italian National Health System were considered in the study; they belong to two rough different sets characterized by either a $300 \mathrm{~s}$ (rtCGM) or 900 s (FGM) sampling period. The FGM set consists of Abbott Freestyle Libre sensors, while the rtCGM group includes Dexcom G4 Platinum, Dexcom G5 Mobile and Medtronic Guardian devices. Each set was assigned to half of the study group according to the center physicians' customary recommendations. Current Italian Standards of Care for Diabetes Management strongly recommend rtCGM for patients with type 1 diabetes and poor glycemic control, especially if hypoglycemia unawareness as well as severe or nocturnal hypoglycemic episodes are present. The latest update also includes specific recommendations of FGM for people with type 1 diabetes whose metabolic control is already adequate but are struggling with frequent glucose testing. When REALISM-T1D started, we did not have specific indications for prescribing FGM with respect to rtCGM. Several elements were then taken into account, including the estimate of individuals' reliability to perform calibrations, their tolerance toward alarms, the interest for future integrated functions of those wearing insulin pumps, and finally patients' own preferences.

\section{Data collection and statistical analysis}

At the end of the observation period all (heterogeneous) raw sensor data were collected from the relevant online repositories (Abbott Libreview, Dexcom Clarity and Glooko-Diasend) and preprocessed to exclude those timeframes where the actual use of the CGM sensor was less than $70 \%,{ }^{22}$ harmonize the data formats, and normalize timestamps with respect to the starting time of monitoring, ${ }^{23}$ so as to make subsequent computeraided analyses easier. This means that the computation of any time point took, respectively, 6000 (FGM) and 18000 (rtCGM) raw sensor data at least. Overall, roughly 6.5 million glucose values were analyzed and processed.

Only continuously stored values were used for FGM sensors, that is, intermittently scanned data were not considered in the study. ${ }^{24}$ Moreover, individuals were constantly reminded and monitored to perform calibrations as specified in the device manufacturer's instructions. Data processing and statistical computation were performed at CNR-IEIIT by means of the GNU Octave scientific programming tool. Although 14 days of CGM data are often considered a good estimation of metrics in a 3-month period, ${ }^{62}$ the TIR percentage was computed using the full 90-day timeframe data to improve both the accuracy and robustness of measurements and reduce noise sensitivity in data acquisition.
In order to quantify the relationship between $\mathrm{A} 1 \mathrm{C}$ and TIR, a linear monovariate regression was conducted on the experimental data set ( $\mathrm{n}=146$ data points) by means of the ordinary least squares (OLS) method, using A1C as predictor and TIR as response variable. On average, each individual contributed 2.5 data points, as 31 measurements out of 177 were discarded because they did not satisfy the ' $70 \%$ CGM use' requirement. The choice of adopting A1C as predictor and TIR as response variable was made because, for general relationship assessment, $\mathrm{A} 1 \mathrm{C}$ values were considered to be reference data, whereas TIR values were subject to measurement noise, which should contribute to the residual OLS variance. This also facilitates the comparison with the results presented in Vigersky and McMahon, ${ }^{17}$ although variables may be swapped, as was done in Bergenstal et $a l^{25}$ where the specific aim is to predict A1C given TIR. For the purpose of the study, TIR percentage calculations were based on an in-range glycemic interval of $70-180 \mathrm{mg} / \mathrm{dL}$, according to recent international consensus of Advanced Technologies \& Treatments of Diabetes (ATTD) experts. ${ }^{6}$ The normality of the residuals and the homogeneity of their variance (homoscedasticity) assumptions were also tested in two alternative ways each. Furthermore, the possible presence of a quadratic component in the regression was assessed by means of a second-degree polynomial OLS. Finally, regression analyses were performed separately for the patient subgroups monitored through either FGM or rtCGM devices, respectively, to assess whether there are statistically significant differences between the two device groups. Statistical significance was evaluated by means of a multivariate OLS analysis with categorical/interaction variables.

\section{RESULTS}

Figure 1 depicts the sample data (" ${ }^{\circ}$ ' markers) and the best-fit regression line. The solid line refers to the interpolation interval from the minimum $\mathrm{A} 1 \mathrm{C}=5.6 \% \quad(38$ $\mathrm{mmol} / \mathrm{mol})$ to the maximum $\mathrm{AlC}=9.2 \%(77 \mathrm{mmol} /$

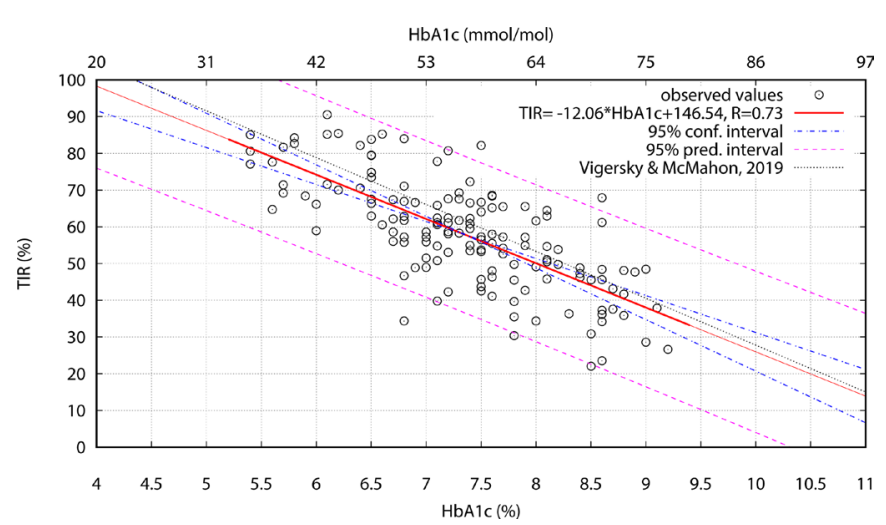

Figure 1 OLS linear regression with 95\% confidence and prediction intervals on the whole data set $(n=146)$, compared with Vigersky and McMahon. ${ }^{17} \beta_{1}=-12.06 \pm 1.86, p<0.001$; $\beta_{0}=146.54 \pm 13.83, p<0.001 ; r^{2}=0.53$. HbA1c, hemoglobin A1c; OLS, ordinary least squares; TIR, time in range. 
mol) observed values. The dot-dashed lines represent the $95 \%$ CI of the best fit, while the dashed lines delimit the $95 \%$ prediction interval. These two intervals are both important because of their two distinct meanings:

- The CI demarcates the region in which the true regression line resides, with a given degree of confidence, and therefore conveys information about the accuracy of the adopted model.

- The prediction interval is the region around an estimated value that contains the corresponding true value with a given degree of confidence. Hence, it expresses the predictive power of the model.

Moreover, the prediction interval considers the residual variance by definition, whereas the CI does not. As a consequence, the prediction interval width also depends, for instance, on the noise introduced by the way the predictor and response variable are measured. This noise may be considered an inherent property of the measurement method, which is therefore independent of the model itself.

The regression coefficients are shown in the figure, while online supplemental appendix tables 1 and 2 list the data sets used in the analysis and the TIR values predicted with the regression separately for the FGM and rtCGM subgroups, besides the experimental observations. The regression points out a strong correlation $\left(\mathrm{r}=0.73, \mathrm{r}^{2}=0.53\right)$ between the predictor and the response variable.

To validate the linear regression model from the mathematical point of view, further tests were conducted to confirm two crucial assumptions, namely the normality of the residuals and the homogeneity of their variance (homoscedasticity). The normality of the residual was successfully confirmed with two distinct methods:

- The quartile-quartile $(\mathrm{Q}-\mathrm{Q})$ plot of the distribution of the residuals, normalized with respect to their estimated variance based on the sample data, versus a normal distribution with zero mean and unity variance, is shown in figure 2A. It reveals an extremely good agreement between the two distributions with only a small number $(<5)$ of outliers at the extremes.

- The Anderson-Darling normality test. ${ }^{26}$ The result of this test, $\mathrm{A}^{2}=0.19$, is well below the critical value, even at a significance level of $10 \%\left(\mathrm{~A}_{0.1}^{2}=0.63\right)$. In addition, the $\mathrm{p}$ value corresponding to the obtained $\mathrm{A}^{2}$ is $0.900,{ }^{27}$ thus giving no reasons to reject the null hypothesis of normality. The Anderson-Darling test was preferred to other, more traditional tests (like the Kolmogorov-Smirnov test) because it is specific to the normal distribution, is independent from the distribution variance, and was found to be very effective in detecting deviations from normality, ${ }^{28}$ and also because it is sensitive to the tails of the distribution under test.

Similarly, the homogeneity of the variances of the residuals was also verified in two distinct ways, one graphical and one analytical:
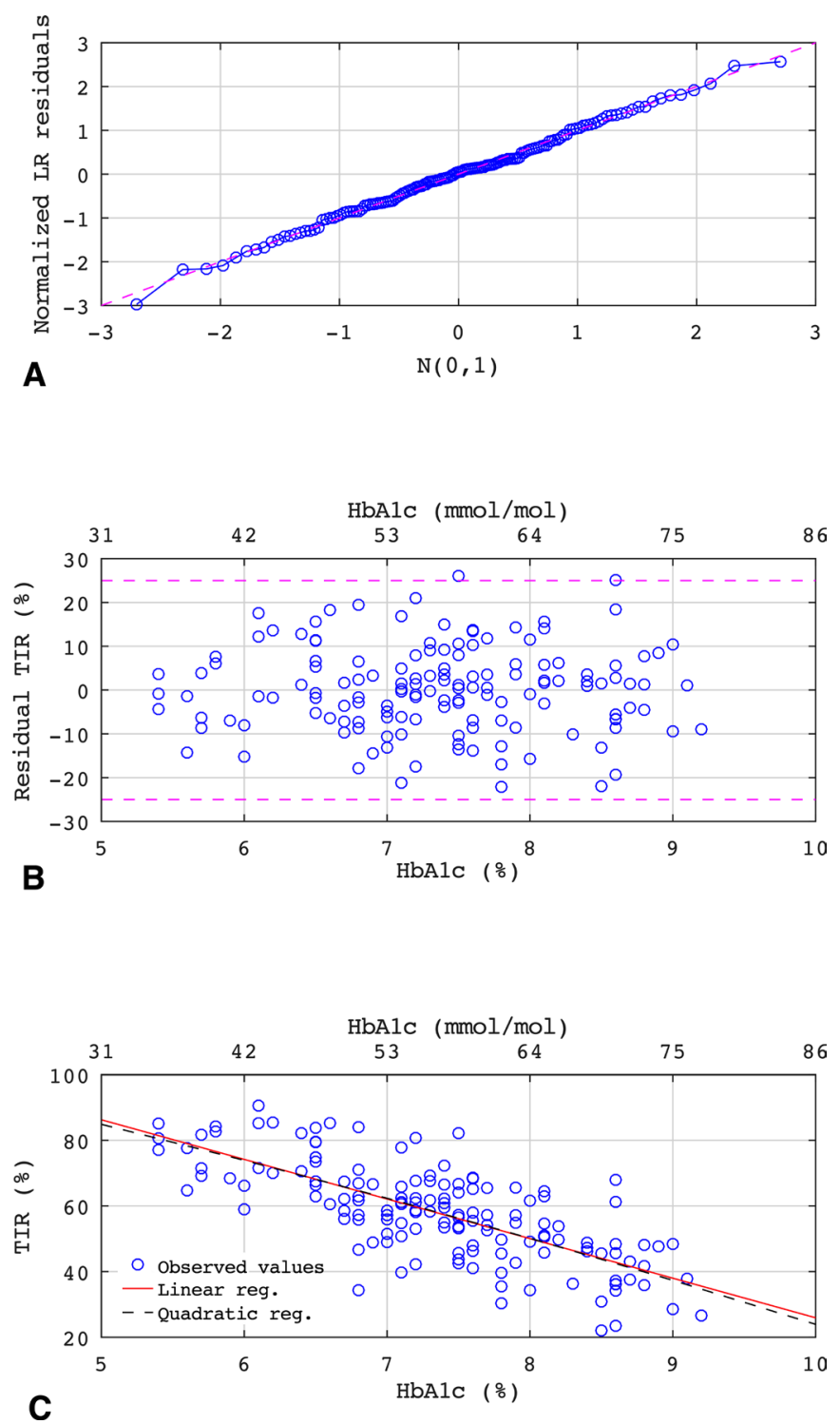

Figure 2 Q-Q plot of normalized residuals versus a normal distribution $\left(\mu=0, \sigma^{2}=1\right)(A)$, scatter plot of the residuals (B), and comparison between a linear and a quadratic OLS regression (C). HbA1c, hemoglobin A1c; LR, linear regression; OLS, ordinary least squares; $\mathrm{Q}-\mathrm{Q}$, quartilequartile; TIR, time in range.

- The scatter plot of the residuals in figure 2B shows that the residuals are contained in the horizontal band \pm 25 , except for three outliers, and there is no visible dependency on the $\mathrm{A} 1 \mathrm{C}$ value in abscissa.

- A standard Goldfeld-Quandt test ${ }^{29}$ was performed by sorting the sample points by increasing values of the predictor and dividing them into three bands. The central band comprising one-third of the samples was then discarded, and two distinct, auxiliary linear regressions were conducted on the remaining two bands. The two sample variances of the residuals $\mathrm{s}_{\mathrm{L}}^{2}=104.400$ (lower band, 49 samples) and $\mathrm{s}_{\mathrm{H}}^{2}=104.488$ (higher band, 49 samples) were then compared with an $F$-test of equality of variances with $(47,47) \mathrm{df}$, obtaining $F=0.999$. The corresponding two-tailed $\mathrm{p}$ 
value, $\mathrm{p}=0.997$, strongly suggests accepting the null hypothesis that the two variances of the residuals are equal.

Another implicit, but equally important hypothesis of linear regression is that the underlying relationship being investigated is indeed linear. In our case, this hypothesis is corroborated by two factors:

- The empirical observation of the scatter plot of the sample data set (figure 1).

- The uniformity of variances (figure 2B and GoldfeldQuandt test) at the extremes of the predictor range, which is in general unlikely to occur when a nonlinear phenomenon is subject to a linear fit.

However, although in the literature some authors advocate a linear relationship, ${ }^{17} 18$ a recent work suggests the presence of a quadratic component. ${ }^{19}$ Therefore, as a further check, we performed an OLS quadratic regression on our sample data. As depicted in figure $2 \mathrm{C}$, which contrasts the two regressions, we noted that in the quadratic regression the magnitude of the quadratic coefficient $\beta_{2}=-0.31$ is negligible because it is more than one order of magnitude smaller than the linear coefficient $\beta_{1}=-7.56$. Moreover, the introduction of the quadratic term did not improve $r^{2}$ in any significant way, and just brought it from $r^{2}=0.5303$ to $r^{2}=0.5307$. We can then conclude that, at least for the study data set, the unexplained variance does not depend on the use of a linear model instead of a quadratic one.

As discussed in the previous section, the TIR of the data set under analysis was computed from measurements taken by two kinds of devices (FGM and rtCGM), which sample the patient's glucose level every $900 \mathrm{~s}$ (and contributed $\mathrm{n}=78$ data points) and $300 \mathrm{~s}$ ( $\mathrm{n}=68$ data points), respectively. To get insights on possible differences between them, two separate linear regressions were performed on the two subsets. The results are presented in figure $3 \mathrm{~A}, \mathrm{~B}$, while sample data are available for reference in online supplemental appendix tables 1 and 2.

The two most important observations concerning this last part of the analysis are the following:

- The $r^{2}$ obtained when considering the first subset of devices (rtCGM with $300 \mathrm{~s}$ sampling interval) is marginally better than the one obtained from the data set as a whole, 0.55 vs 0.53 , and the regression line exhibits a better agreement with the results of Vigersky and McMahon. ${ }^{17}$

- Restricting the analysis to data collected from devices with a 900 s sampling interval (FGM devices) results instead in a deterioration of $\mathrm{r}^{2}$, from 0.53 to 0.49 , as well as a departure from the results of Vigersky and McMahon. ${ }^{17}$

The statistical significance of the observed difference was tested by means of a multivariate OLS on all samples (from both rtCGM and FGM devices) in which, besides A1C, a dichotomous categorical variable $c$ ( 0 for rtCGM and 1 for FGM samples) and an interaction variable $d=$ A1C. $c$ were introduced. ${ }^{30}$ The coefficients of $c$ and $d$ quantify the intercept and slope difference, respectively,
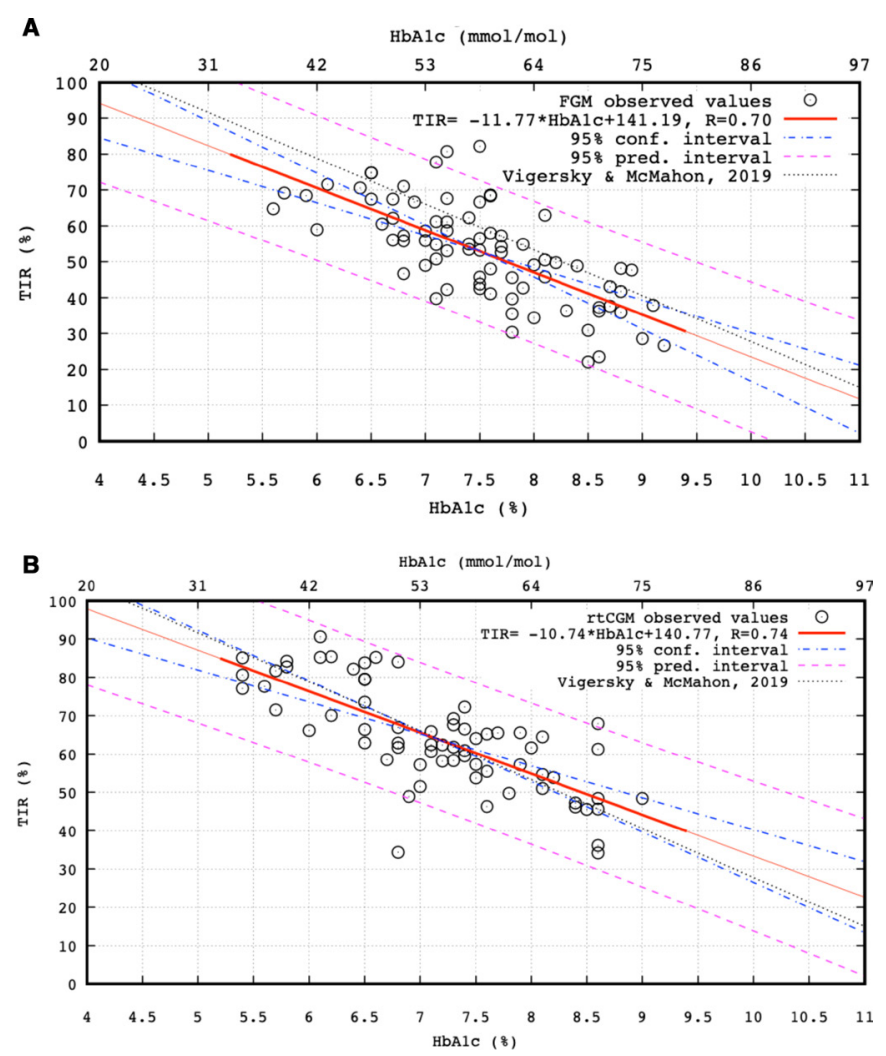

Figure 3 Linear regressions performed separately on patient groups monitored with different device types (FGM or rtCGM) and different sampling periods (900 s or $300 \mathrm{~s}$ ) compared with Vigersky and McMahon. ${ }^{17}$ (A) FGM group: $\beta_{1}=-11.77 \pm 2.73, p<0.001 ; \beta_{0}=141.19 \pm 20.6, p<0.001 ; r^{2}=0.49$. (B) rtCGM group: $\beta_{1}=-10.74 \pm 2.4, p<0.001 ; \beta_{0}=140.77 \pm 17.35$, $\mathrm{p}<0.001 ; r^{2}=0.55$. CGM, continuous glucose monitoring; FGM, flash CGM; HbA1c, hemoglobin A1c; rtCGM, real-time CGM; TIR, time in range.

while their two-tailed $p$ values indicate whether the difference is significant or not. The results $(\mathrm{p}<0.001$ for $c$ and $\mathrm{p}=0.57$ for $d$ ) show that there is a statistically significant difference between the intercepts, given by $c$ 's coefficient $\beta_{c}=-7.19$, but not between the slopes. Furthermore, the introduction of $c$ increased $\mathrm{r}^{2}$ from 0.53 to 0.59 and decreased the sample variance of the residuals from $\mathrm{s}^{2}{ }_{\mathrm{NC}}=102.335$ to s${ }_{\mathrm{C}}^{2}=89.921$. An $F$-test of equality of these variances with $(144,143) \mathrm{df}$ gave $F=1.135$ and a two-tailed $\mathrm{p}=0.22$. Figure 4 illustrates graphically how the introduction of $c$ (on the y axis) affected the regression and improved the fit of TIR ( $\mathrm{z}$ axis) as a function of A1C ( $\mathrm{x}$ axis).

\section{CONCLUSIONS}

The careful assessment of the TIR-A1C relationship in real-life conditions is important for diabetologists to gain confidence and rely on this and other CGM-derived metrics in their usual practice. Differently from other works based on randomized studies, such as Vigersky and McMahon, ${ }^{17}$ Beck et al, ${ }^{18}$ and Petersson et al, ${ }^{19}$ our investigations were based on pure 1-year observations of 70 patients with type 1 diabetes adopting CGM in 


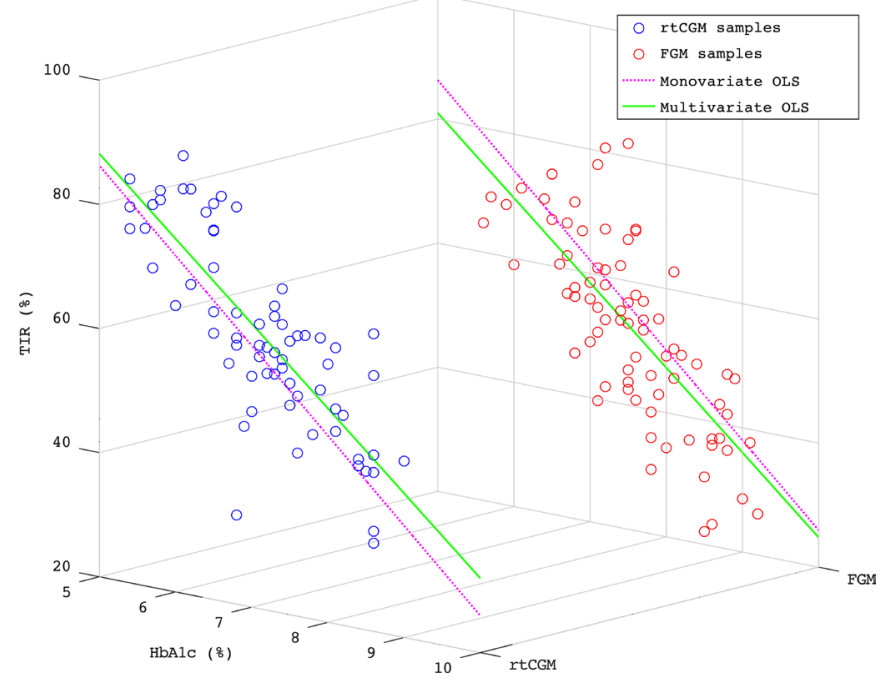

Figure 4 Monovariate versus multivariate regression using a categorical variable $c$ to indicate FGM or rtCGM devices. Categorical coefficient $\beta=-7.19 \pm 3.20, p<0.001$. CGM, continuous glucose monitoring; FGM, flash CGM; HbA1c, hemoglobin A1c; OLS, ordinary least squares; rtCGM, realtime CGM; TIR, time in range.

their everyday routine. TIR percentages were evaluated directly from the raw sensor data over long (90 days) periods of at least $70 \%$ CGM use, as opposite to average values derived from larger populations but in more heterogeneous conditions, including for instance both type 1 and type 2 diabetes, juvenile and/or adult patients, blood glucose monitoring, and different and shorter CGM durations (eg, from 7 to 84 days in Vigersky and McMahon, ${ }^{17} 6$ months in Beck et al, ${ }^{18}$ and 60 days in Petersson et $a l^{19}$ ) and registration times. Results obtained in this way confirm a linear relationship between TIR and $\mathrm{A} 1 \mathrm{C}$ as quadratic contributions, unlike in Petersson et $a l,{ }^{19}$ were found to be negligible. Values of the regression slope and intercept obtained for the whole data set are similar to results from Vigersky and McMahon, ${ }^{17}$ with a slightly weaker correlation in our case. This can be partially justified considering that Vigersky and McMahon $^{17}$ sourced selected paired (hemoglobin A1c, TIR) metrics from multiple articles. Hence, the averaging process used in those articles to calculate the paired metrics may have had a damping effect on the residual variance of the subsequent linear regression analysis, with respect to the direct use of the original, more dispersed data points. As each REALISM-T1D individual contributed to the study with 2.5 data points, on average, distinct linear regressions were also computed separately using points collected at $\mathrm{t}=90, \mathrm{t}=180$ and $\mathrm{t}=365$, respectively (where each individual contributed a single point), to investigate possible correlation effects in the pooled analysis. Results reported in figure 5 show that differences in the $6 \%-9 \%$ A1C range are quite limited for the three time-instants considered and compatible with typical individual glucose variations over a 90-day time interval, while $\mathrm{r}$ always remains greater than 0.72 .
Consequently, the pooled analysis does not seem to be affected by correlation problems due to multiple point contributions by the same individual.

More evident differences exist between patients who were prescribed either an FGM or rtCGM sensor. In the first case, the computed regression coefficients are still close to those presented in Vigersky and McMahon, ${ }^{17}$ although $r$ is lower. When rtCGM devices are considered, instead, the distance of $\beta_{1}$ and $\beta_{0}$ with respect to Vigersky and McMahon ${ }^{17}$ increases, but $r$ improves. This difference in behavior also becomes evident by comparing figure 3A,B. In our opinion this may be due to the heterogeneity of conditions and devices in Vigersky and McMahon $^{17}$ and partly in figure $3 \mathrm{~A}$, which tends to mask diversities in the overall regression computation. Differences become clearer when homogeneous groups are considered, such as in figure 3A,B. This also stresses the need for the availability of more data and investigations if TIR and other CGM-related metrics have to be massively adopted in routine practice, since a set of 'good for all' parameters values, such as for the joint FGM and rtCGM group, can hardly meet individual patient conditions.

In fact, a $0.5 \%$ decrease in $\mathrm{A} 1 \mathrm{C}$, from $7.5 \%$ (58 mmol/ $\mathrm{mol}$ ) to $7.0 \%$ (53 mmol $/ \mathrm{mol})$, means an improvement of the predicted TIR percentage from $52.9 \%$ to $58.8 \%$ for the FGM group and from $60.2 \%$ to $65.6 \%$ for the rtCGM group. Although the absolute variation is similar $(5.9 \%$ vs $5.4 \%$ ), the impact is significantly different in the two situations because of the role played by the corresponding values of $\beta_{1}$ and $\beta_{0}$, which match the same $A 1 C$ value with higher TIR percentages for the rtCGM group than for FGM. The unavailability of CGM (TIR) data at baseline cannot exclude for sure that this discrepancy might be caused by some bias effect in the study, despite no appreciable differences existing in the two groups concerning anthropometry and therapy. An additional check carried out in the regression analysis by dropping two sensorsampled data out of three in the rtCGM case (thus simulating a lower sampling rate equal to the FGM sensors) resulted in no appreciable changes in the computed TIR

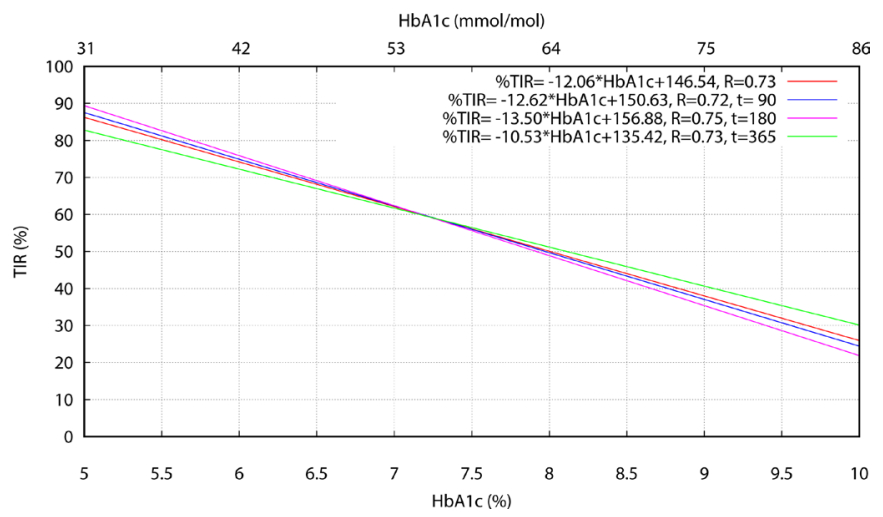

Figure 5 Linear regressions performed separately at time points $t=90$ days, $t=180$ days and $t=365$ days using both FGM and rtCGM sensor data. CGM, continuous glucose monitoring; FGM, flash CGM; HbA1c, hemoglobin A1c; rtCGM, real-time CGM; TIR, time in range. 
values and consequently in the regression coefficients. Other reasons, possibly deserving more investigations, are differences in sensor technology and patient-induced behavior. Actually, the FGM sensors used in the study are unable to generate alerts and alarms for hypoglycemia and hyperglycemia, likely requiring more attention and intervention by patients, thus justifying slower reaction times, which ultimately can result in a reduction in the overall time spent in the $70-180 \mathrm{mg} / \mathrm{dL}$ range.

The power of A1C as a predictor of TIR is another aspect of utmost interest and, to some extent, concern. The rather large prediction interval boundaries depicted in figure $3 \mathrm{~A}$ show that even in the central zone around $\mathrm{A} 1 \mathrm{C}=7.5 \%(58 \mathrm{mmol} / \mathrm{mol})$, where the uncertainty of the predicted TIR $=56 \%$ is the smallest, the true TIR value can actually vary from $34 \%$ to $78 \%$. It is worth noting that these figures are in good accordance with those reported in Beck et $a l^{18}$ where a CI of 38 to 79 was established for a TIR predicted value equal to $59 \%$. When FGM and rtCGM groups are considered, the predicted values for $\mathrm{A} 1 \mathrm{C}=7.5 \%(58 \mathrm{mmol} / \mathrm{mol})$ are $\mathrm{TIR}=54 \%$ and $\mathrm{TIR}=60 \%$, respectively, while the prediction interval is 33-73 in the former case and 42-78 in the latter, which imply a maximum relative difference between true and estimated values of about $37 \%$ for FGM and 30\% for rtCGM. In turn, this highlights possible limitations of A1C in predicting the TIR percentage accurately and confirms the opportunity of having different models for different patient groups as suggested by some authors, ${ }^{31}$ as well as for FGM versus rtCGM devices, due to the statistically significant difference between their OLS regression models.

Acknowledgements The University of Torino and the Division of Endocrinology and Metabolic Diseases at Azienda Ospedaliera Universitaria Città della Salute e della Scienza di Torino supported the observation, monitoring and follow-up of patients in this study. All CGM devices used in the study were prescribed and reimbursed by the Italian National Health System. The Institute of Electronics, Information and Telecommunication Engineering of the National Research Council of Italy supported the data processing and analysis. All institutions evenly supported the writing of the manuscript.

Contributors MV and GG designed the study and took care of patient follow-up and also supervised all visits. ICB and AV performed the relevant data processing and statistical analysis. All authors contributed significantly to the writing and editing process of this manuscript. GG is the guarantor of this work and as such had full access to all the data in the study and takes responsibility for the integrity of the data and the accuracy of the data analysis.

Funding The authors have not declared a specific grant for this research from any funding agency in the public, commercial or not-for-profit sectors.

Competing interests None declared.

Patient consent for publication Not required.

Ethics approval All procedures performed in this study involving human participants were in accordance with the authors' institutions' ethical recommendations and with the 1964 Helsinki Declaration and its later amendments.

Provenance and peer review Not commissioned; externally peer reviewed.

Data availability statement All data relevant to the study are included in the article or uploaded as supplemental information.

Supplemental material This content has been supplied by the author(s). It has not been vetted by BMJ Publishing Group Limited (BMJ) and may not have been peer-reviewed. Any opinions or recommendations discussed are solely those of the author(s) and are not endorsed by BMJ. BMJ disclaims all liability and responsibility arising from any reliance placed on the content. Where the content includes any translated material, BMJ does not warrant the accuracy and reliability of the translations (including but not limited to local regulations, clinical guidelines, terminology, drug names and drug dosages), and is not responsible for any error and/or omissions arising from translation and adaptation or otherwise.

Open access This is an open access article distributed in accordance with the Creative Commons Attribution Non Commercial (CC BY-NC 4.0) license, which permits others to distribute, remix, adapt, build upon this work non-commercially, and license their derivative works on different terms, provided the original work is properly cited, appropriate credit is given, any changes made indicated, and the use is non-commercial. See: http://creativecommons.org/licenses/by-nc/4.0/.

\section{ORCID iDs}

Marina Valenzano http://orcid.org/0000-0003-3484-4626

Ivan Cibrario Bertolotti http://orcid.org/0000-0002-9607-9993

Adriano Valenzano http://orcid.org/0000-0003-1238-0808

Giorgio Grassi http://orcid.org/0000-0001-8201-4219

\section{REFERENCES}

1 Rodbard D. Continuous glucose monitoring: a review of successes, challenges, and opportunities. Diabetes Technol Ther 2016;18:S23-S2-13.

2 Wood A, O'Neal D, Furler J, et al. Continuous glucose monitoring: a review of the evidence, opportunities for future use and ongoing challenges. Intern Med J 2018;48:499-508.

3 Ekhlaspour L, Tabatabai I, Buckingham B. A review of continuous glucose monitoring data interpretation in the age of automated insulin delivery. J Diabetes Sci Technol 2019;13:645-63.

4 Cappon G, Acciaroli G, Vettoretti M. Wearable continuous glucose monitoring sensors: a revolution in diabetes treatment. Electronics 2017:65:1-16.

5 Cappon G, Vettoretti M, Sparacino G, et al. Continuous glucose monitoring sensors for diabetes management: a review of technologies and applications. Diabetes Metab J 2019;43:383.

6 Battelino T, Danne T, Bergenstal RM, et al. Clinical targets for continuous glucose monitoring data interpretation: recommendations from the International consensus on time in range. Diabetes Care 2019;42:1593-603.

7 Johnson ML, Martens TW, Criego AB, et al. Utilizing the ambulatory glucose profile to standardize and implement continuous glucose monitoring in clinical practice. Diabetes Technol Ther 2019;21:S217-S2-25.

8 Beyond A1C Writing Group. Need for regulatory change to incorporate beyond A1c glycemic metrics. Diabetes Care 2018;41:e92-4.

9 Wright LA-C, Hirsch IB. Metrics beyond hemoglobin A1C in diabetes management: time in range, hypoglycemia, and other parameters. Diabetes Technol Ther 2017;19:S-16-S-26.

10 Chehregosha $\mathrm{H}$, Khamseh ME, Malek M, et al. A view beyond HbA1c: role of continuous glucose monitoring. Diabetes Ther 2019;10:853-63.

11 Tylee TS, Trence DL. Glycemic variability: looking beyond the A1C. Diabetes Spectrum 2012;25:149-53.

12 Umpierrez GE, P Kovatchev B. Glycemic variability: how to measure and its clinical implication for type 2 diabetes. Am J Med Sci 2018;356:518-27.

13 Lee J-E. Alternative biomarkers for assessing glycemic control in diabetes: fructosamine, glycated albumin, and 1,5-anhydroglucitol. Ann Pediatr Endocrinol Metab 2015;20:74-8.

14 Beck RW, Connor CG, Mullen DM, et al. The Fallacy of Average: How Using $\mathrm{HbA}_{1 \mathrm{c}}$ Alone to Assess Glycemic Control Can Be Misleading. Diabetes Care 2017;40:994-9.

15 Garg SK, Akturk HK. The future of continuous glucose monitoring. Diabetes Technology and Therapeutics 2017;10:S1-2.

16 Allen N, Gupta A. Current diabetes technology: striving for the artificial pancreas. Diagnostics 2019;9:31.

17 Vigersky RA, McMahon C. The relationship of hemoglobin A1c to time-in-range in patients with diabetes. Diabetes Technol Ther 2019;21:81-5.

18 Beck RW, Bergenstal RM, Cheng P, et al. The relationships between time in range, hyperglycemia metrics, and HbA1c. J Diabetes Sci Technol 2019;13:614-26.

19 Petersson J, Åkesson K, Sundberg F, et al. Translating glycated hemoglobin A1c into time spent in glucose target range: a multicenter study. Pediatr Diabetes 2019;20:339-44.

20 Dunn TC, Xu Y, Hayter G, et al. Real-World flash glucose monitoring patterns and associations between self-monitoring frequency and 
glycaemic measures: a European analysis of over 60 million glucose tests. Diabetes Res Clin Pract 2018;137:37-46.

21 Blonde L, Khunti K, Harris SB, et al. Interpretation and impact of real-world clinical data for the practicing clinician. Adv Ther 2018;35:1763-74.

22 Riddlesworth TD, Beck RW, Gal RL, et al. Optimal sampling duration for continuous glucose monitoring to determine long-term glycemic control. Diabetes Technol Ther 2018;20:314-6.

23 Petrie JR, Peters AL, Bergenstal RM, et al. Improving the clinical value and utility of CGM systems: issues and recommendations : A joint statement of the European Association for the Study of Diabetes and the American Diabetes Association Diabetes Technology Working Group. Diabetologia 2017;60:2319-28.

24 Pleus S, Kamecke U, Link M, et al. Flash glucose monitoring: differences between intermittently scanned and continuously stored data. J Diabetes Sci Technol 2018;12:397-400.
25 Bergenstal RM, Beck RW, Close KL, et al. Glucose management indicator (GMI): a new term for estimating A1c from continuous glucose monitoring. Diabetes Care 2018;41:2275-80.

26 Anderson TW, Darling DA. Asymptotic Theory of Certain "Goodness of Fit" Criteria Based on Stochastic Processes. Ann Math Statist 1952;23:193-212.

27 D'Agostino RB, A: Stephens M, eds. Goodness-of-Fit Techniques. Marcel Dekker, 1986

28 Stephens MA. Edf statistics for Goodness of fit and some comparisons. J Am Stat Assoc 1974;69:730-7.

29 Goldfeld SM, Quandt RE. Some tests for Homoscedasticity. J Am Stat Assoc 1965;60:539-47.

30 Bishop YM, Fienberg SE. Discrete multivariate analysis: theory and practice. MIT Press, 1974.

31 Dixon RF, Miller DP, Majithia A, et al. 105-LB: does HbA1c accurately predict time-in-range? Diabetes 2019;68:105-LB. 